\title{
MicroRNA-204 may participate in the pathogenesis of hypoxic-ischemic encephalopathy through targeting KLLN
}

\author{
RONGLIN CHEN ${ }^{1}$, MEIXIA WANG ${ }^{1}$, SHAOPIN FU $^{1}$, FENG CAO $^{1}$, PENGKAI DUAN $^{2}$ and JIEFU LU ${ }^{2}$ \\ ${ }^{1}$ Department of Critical Care Medicine, Longgang District Central Hospital, Shenzhen, Guangdong 518116; \\ ${ }^{2}$ Department of Intensive Care Unit, Affiliated General Hospital of Guangzhou Military Command of \\ Southern Medical University, Guangzhou, Guangdong 510515, P.R. China
}

Received March 15, 2018; Accepted November 14, 2018

DOI: $10.3892 / \mathrm{etm} .2019 .7936$

\begin{abstract}
Hypoxic-ischemic encephalopathy (HIE) is a common neonatal disease that can lead to high neonatal mortality rates. Previous studies have indicated that microRNAs (miRs) may be involved in the pathogenesis of HIE; however, the specific mechanisms underlying their involvement require further investigation. The aim of the present study was to investigate the roles of miR-204 and its target gene killin p53 regulated DNA replication inhibitor (KLLN) in HIE using rat HIE models. Brain injury was induced by surgery and incubation of hypoxic incubator brain using 10-day-old pup rats. On day 3 , rats were sacrificed, and the infarct size of the brain was determined using a tetrazolium chloride assay. Terminal deoxynucleotidyl transferase UTP nick-end labeling staining was performed to detect the cell death rate in the brain tissue. Following this, the brain tissues were collected, and reverse transcription-quantitative polymerase chain reaction, western blot analysis and immunohistochemistry assays were performed to examine the expression levels of miR-204 and KLLN. Furthermore, neurons were cultured and transfected with miR-204 inhibitors or mimics, and the effect of miR-204 on the proliferation and apoptosis of neurons was examined using MTT and flow cytometric assays. Finally, a dual-luciferase reporter assay was performed to confirm whether KLLN is a direct target of miR-204. The expression of miR-204 was significantly downregulated and the expression of KLLN was significantly increased in the brain tissue of HIE rats $(\mathrm{P}<0.001)$. In addition, the transfection with miR-204 inhibitors significantly decreased the proliferation rates and significantly increased the apoptosis rate of neurons; however, transfection with miR-204 mimics prompted the opposite results. The dual-luciferase
\end{abstract}

Correspondence to: Dr Ronglin Chen, Department of Critical Care Medicine, Longgang District Central Hospital, 6082 Longgang Avenue, Shenzhen, Guangdong 518116, P.R. China

E-mail: dr_Ronglinchen@outlook.com

Key words: microRNA-204, killin p53 regulated DNA replication inhibitor, hypoxic-ischemic encephalopathy, pathogenesis reporter assay also confirmed that KLLN is a direct target of miR-204. Taken together, the results of the present study demonstrated that miR-204 was downregulated in HIE and that miR-204 may serve important roles in the pathogenesis of HIE through targeting KLLN.

\section{Introduction}

Perinatal hypoxic-ischemic encephalopathy (HIE) is a common neonatal malignancy (1). HIE can lead to high neonatal mortality rates and causes long-term severe neurologic morbidity (2). Unfortunately, the pathogenesis of HIE remains unclear, leading to poor prognosis and severe sequelae (3). Currently, therapeutic hypothermia is the most common treatment for moderate to severe HIE; however, the efficacy is unsatisfactory, and $\sim 50 \%$ of infants succumb or suffer from neurological disabilities following treatment with hypothermia (4). Thus, there is an urgent requirement to investigate the underlying mechanisms and identify novel therapeutic targets for the treatment of HIE.

In recent years, increasing evidence has indicated that microRNAs (miRs) may participate in the pathogenesis of various neurological disorders, including ischemic stroke (5). miRs are small non-coding RNAs, 21-22 nucleotides in length, that bind to the 3'-untranslated region (3'-UTR) of their target mRNAs, which can silence the target mRNAs and lead to translational suppression (6). Previous studies have indicated that miRs are involved in various important cellular events, including the proliferation, differentiation, apoptosis and migration of cells (7-10). Furthermore, the roles of miRs in the pathogenesis of different diseases have also been explored (11). In the field of neuroscience, it has been confirmed that miRs are enriched in the nervous system and serve key roles in the development of brain.

Previous studies have indicated that the expression of miR-204 is aberrantly expressed in tissue samples and the peripheral blood from patients and animal models of HIE $(12,13)$; however, the specific mechanism remains to be elucidated. In the present study, the roles of miR-204 and its target gene killin p53 regulated DNA replication inhibitor (KLLN) were investigated in HIE using rat HIE models. This study may provide a novel therapeutic target for the treatment of HIE. 


\section{Materials and methods}

Establishing a neonatal rat HIE model. The Rice-Vannucci model was established as described previously (13). Briefly, a total of 20 neonatal Sprague-Dawley rat pups (age, 10 days; weight, $12.5-15.0 \mathrm{~g}$ ) were purchased from the animal center of Nanjing Medical University (Nanjing, China). Rats were maintained at the atmosphere of $55 \pm 5 \%$ with a temperature of $25 \pm 2^{\circ} \mathrm{C}$ on a 12 -h light/dark cycle and the food and water were provided ad libitum. The unsexed neonatal rat pups were randomly divided into either the sham $(\mathrm{n}=10)$ or HIE model $(n=10)$ group. In the HIE model group, rat pups were anesthetized and the right common carotid artery (CCA) was exposed, followed by double ligation with 5.0 silk surgical suture. Following surgery, rats were allowed to recuperate for $1 \mathrm{~h}$, and then placed into a humidified hypoxic incubator containing $8 \%$ oxygen balanced with $92 \%$ nitrogen at $37^{\circ} \mathrm{C}$ for $1.5 \mathrm{~h}$. For the sham group, the CCA of the rats was exposed, without any ligation or hypoxic treatment (14). Following surgery, rat pups were maintained at $32^{\circ} \mathrm{C}$ with a 12 -h light/dark cycle for $48 \mathrm{~h}$. All procedures were approved by the Ethics Committee of Longgang District Central Hospital (Shenzhen, China).

Brain infarct size measurement. The infarct size of the brain was determined using 2,3,5-triphenyltetrazolium chloride monohydrate (TTC; Sigma-Aldrich; Merck KGaA, Darmstadt, Germany) staining, as described previously (15). Briefly, 48-h after ligation and hypoxic treatment, rat pups were anesthetized, and the head and neck of the rats were cut and the skull of the rats were stripped to expose the cerebral tissue. Next, the entire brain tissue was removed and serial 2-mm thick coronal sections of the brain were cut and immersed into TTC solution (2\%) for $5 \mathrm{~min}$ at $37^{\circ} \mathrm{C}$. Subsequently, brain sections were fixed with $10 \%$ formaldehyde at room temperature overnight. The caudal and the rostral surfaces of each slice were imaged using a light microscope (magnification, 200x; Olympus IX70; Olympus Corporation, Tokyo, Japan).

Terminal deoxynucleotidyl transferase UTP nick-end labeling (TUNEL) assay. TUNEL staining was performed to detect the cell death rate in the brain tissue according to manufacturer's protocol. Briefly, the brain tissue sections were fixed with paraformaldehyde $(40 \mathrm{~g} / \mathrm{l})$ for $24 \mathrm{~h}$ at room temperature and subsequently incubated with TUNEL reagent at $37^{\circ} \mathrm{C}$ for $1 \mathrm{~h}$ in the dark. Following incubation, cells were washed three times with PBS. Cell nuclei were counterstained with $1 \mu \mathrm{g} / \mathrm{ml}$ DAPI for $5 \mathrm{~min}$ at room temperature and mounted in anti-fade mounting medium (cat. no. P0126; Beyotime Institute of Biotechnology). TUNEL-positive cells were observed in four randomly-selected fields under a Zeiss LSM 710 confocal microscope (magnification 20x; Carl Zeiss Ag, Oberkochen, Germany). TUNEL-positive cells in the cortex or the hippocampus in the penumbra were quantified using ImageJ software (version 1.8; National Institutes of Health, Bethesda, MD, USA). The nucleus counter particle analysis plug-in was used for cell counting.

Cell culture. Primary rat hippocampus neurons (cat. no. A1084101) were purchased from Thermo Fisher Scientific, Inc. (Waltham, MA, USA). Cells were cultured in RPMI-1640
A
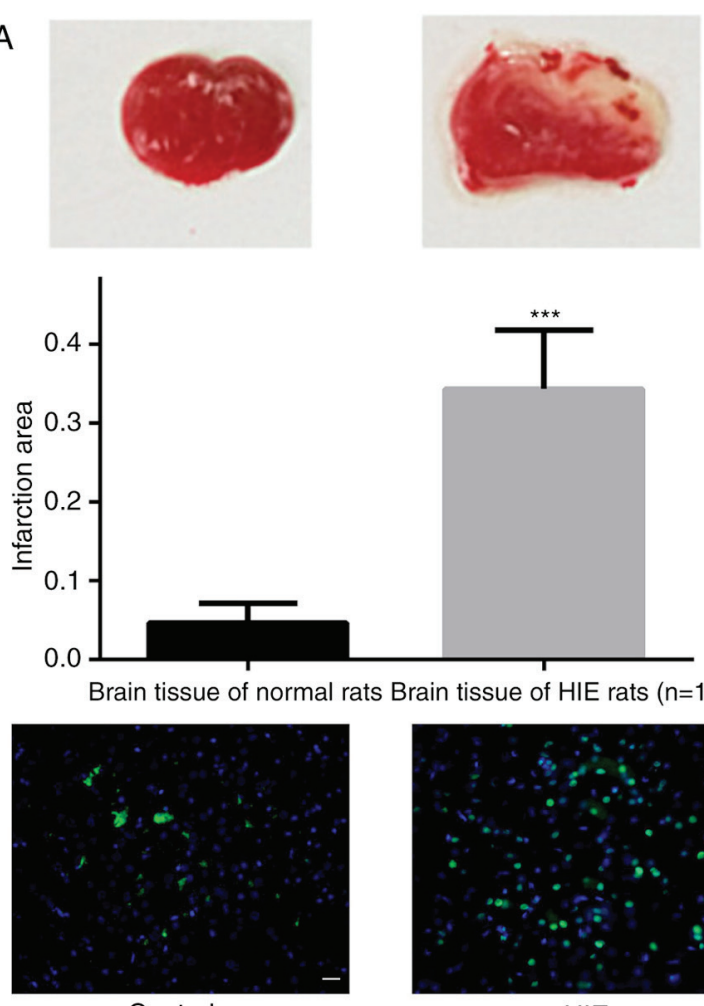

Control

C

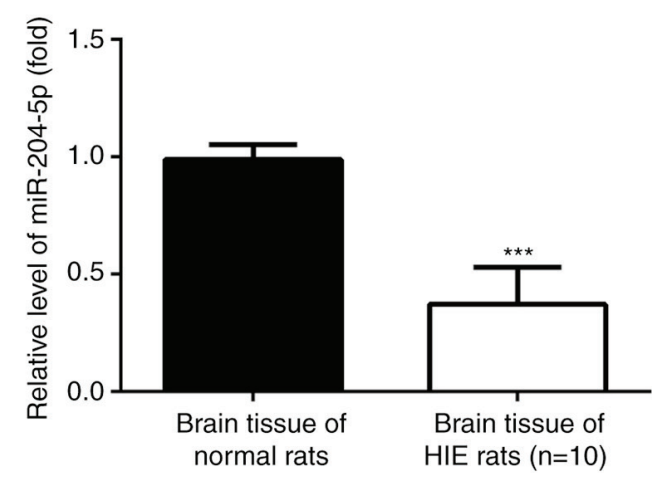

Figure 1. Establishing of the HIE models. (A) The 2,3,5-triphenyltetrazolium chloride monohydrate staining results of the normal brain and HIE brain (magnification, 200x). (B) Results of terminal deoxynucleotidyl transferase UTP nick-end labeling staining of the normal brain and HIE brain (scale bar, $100 \mu \mathrm{m})$. (C) Relative expression of miR-204 in the brain tissue of HIE rats. ${ }^{* * * *} \mathrm{P}<0.001$ vs. normal rats. HIE, hypoxic-ischemic encephalopathy; miR, microRNA.

(Thermo Fisher Scientific, Inc.) supplemented with $10 \%$ fetal bovine serum (Thermo Fisher Scientific, Inc.), $100 \mathrm{U}$ penicillin $/ \mathrm{ml}$ and $100 \mathrm{mg}$ streptomycin $/ \mathrm{ml}$ and maintained $37^{\circ} \mathrm{C}$ in a $5 \% \mathrm{CO}_{2}$-humidifed incubator.

Cell transfection. miR-204 mimics, miR-204 mimics negative control (NC), miR-204 inhibitor and miR-204 NC oligonucleotides were purchased from GenePharma (Shanghai, China). Neurons were seeded into 6-well plates at a density of $\sim 1 \times 10^{5}$ cells/well. Cells were subsequently transfected with $50 \mathrm{nM}$ miR-204 mimics (5'-UUCCCUUUGUCAUCCUAU GCCU-3'), miR-204 mimics NC (5'-UUUGUACUACACAAA AGUACUG-3'), miR-204 inhibitor (5'-AGGATGACAAAG GGA-3') or miR-204 inhibitor NC (5'-ACGTCTATACGC CCA- 3') using Lipofectamine RNAiMax transfection reagent 
miR-204 inhibitor

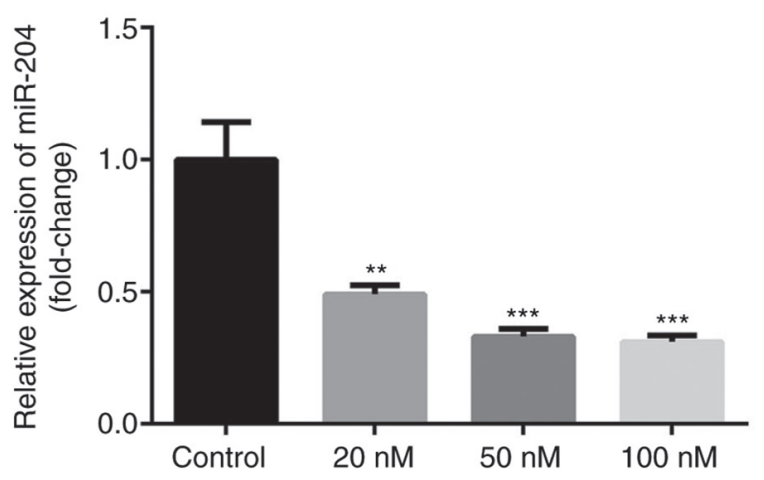

miR-204 mimics

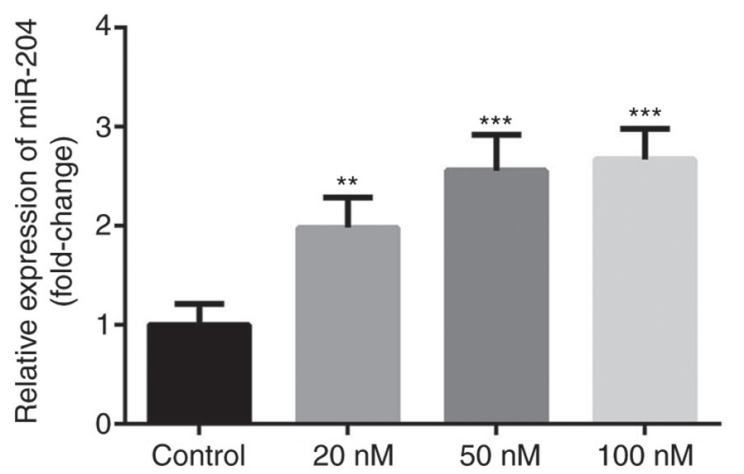

miR-204 inhibitor NC

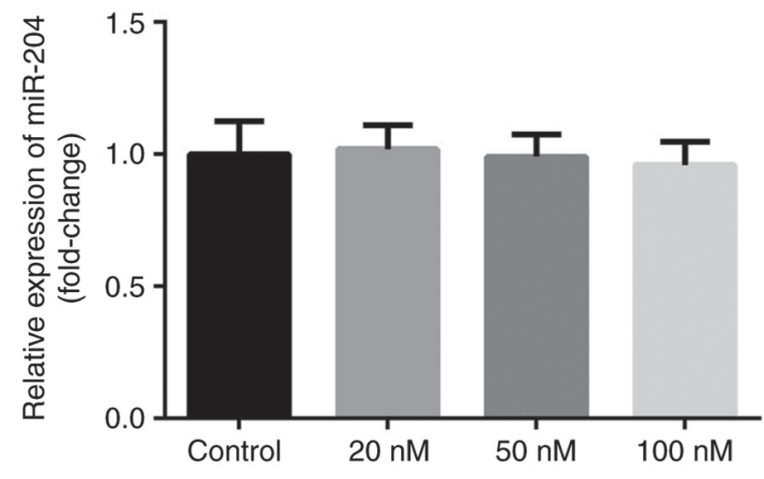

miR-204 mimics NC

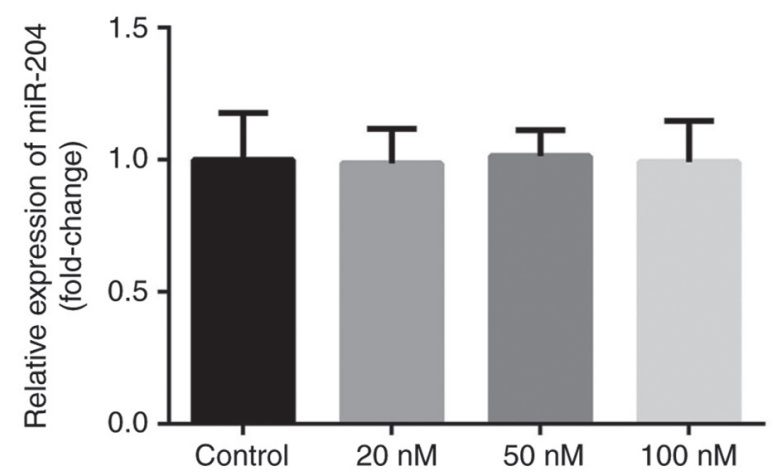

Figure 2. Effect of miR-204 mimics or inhibitor on the expression of miR-204 in neurons. Reverse transcription-quantitative polymerase chain reaction analysis was performed to assess miR-204 expression. ${ }^{* *} \mathrm{P}<0.01$ and ${ }^{* * * *} \mathrm{P}<0.001$ vs. control. Control, untransfected cells; miR-204, microRNA-204; NC, negative control; miR-204 inhibitor, cells transfected with miR-204 inhibitor; miR-204 inhibitors NC, cells transfected with miR-204 inhibitor NC; miR-204 mimics, cells transfected with miR-204 mimics; miR-204 mimics NC, cells transfected with miR-204 mimics NC.

(Thermo Fisher Scientific, Inc.). Cells were harvested for subsequent analysis following 48-h incubation.

Reverse transcription-quantitative polymerase chain reaction $(R T-q P C R)$. Total RNA was extracted from brain tissue samples using TRIzol ${ }^{\circledR}$ reagent (Invitrogen; Thermo Fisher Scientific, Inc.). To examine KLLN mRNA levels, RT-qPCR was performed using the One Step TB Green ${ }^{\circledR}$ PrimeScript $^{\mathrm{TM}}$ RT-PCR kit (Perfect Real Time) (Takara Biotechnology Co., Ltd., Dalian, China) on an ABI 7300 Real-Time PCR system (Applied Biosystems; Thermo Fisher Scientific, Inc.), according to manufacturer's protocol. To examine miR-204 levels, qPCR was performed using the Hairpin-it miRNAs qPCR Quantitation kit (GenePharma, Shanghai, China), according to the manufacturer's protocol. Primers were synthesized by Genscript Nanjing Inc. (Nanjing, China) and the following primer pairs were used for RT-qPCR: miR-204 forward, 5'-GCGGCGCAAAGAATTCTCCT-3' and reverse, 5'-GTGCAGGGTCCGAGGT-3'; U6 forward, 5'-CTCGCT TCGGCAGCACA-3' and reverse, 5'-AACGCTTCACGAATT TGCGT-3'; KLLN forward, 5'-ATGGATCGCCCGGGGCCA G-3' and reverse, 5'-TCAGTCCTTTGGCTTGCTCTTA-3'; GAPDH forward, 5'-TTGATGGCAACAATCTCCAC-3' and reverse 5'-CGTCCCGTAGACAAAATGGT-3'. The following thermocycling conditions were used for RT-qPCR: Initial denaturation at $95^{\circ} \mathrm{C}$ for $30 \mathrm{sec} ; 40$ cycles of $95^{\circ} \mathrm{C}$ for $5 \mathrm{sec}$ and $60^{\circ} \mathrm{C}$ for $30 \mathrm{sec}$. KLLN and miR-204 levels were quantified using

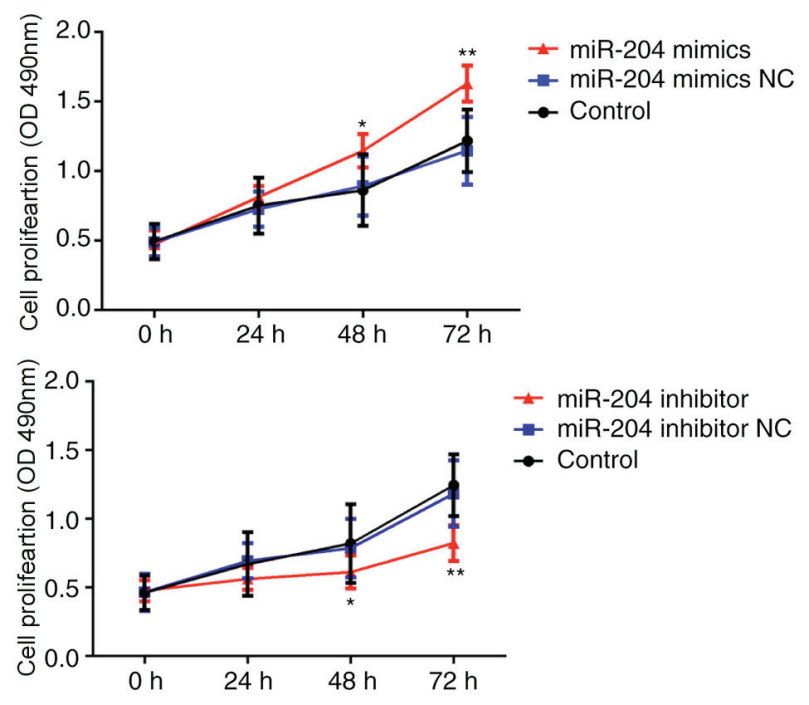

Figure 3. Effect of miR-204 on the proliferation of neurons. MTT assays were performed on neurons at $0,24,48$ and $72 \mathrm{~h}$. ${ }^{*} \mathrm{P}<0.05$ and ${ }^{* *} \mathrm{P}<0.01$ vs. control. Control, untransfected cells; OD, optical density; miR-204, microRNA-204; NC, negative control; miR-204 inhibitor, cells transfected with miR-204 inhibitor; miR-204 inhibitors NC, cells transfected with miR-204 inhibitor NC; miR-204 mimics, cells transfected with miR-204 mimics; miR-204 mimics NC, cells transfected with miR-204 mimics NC.

the $2^{-\Delta \Delta C q}$ method (16) and normalized to the internal reference gene GAPDH or U6 (RNU6B; GenePharma), respectively. 

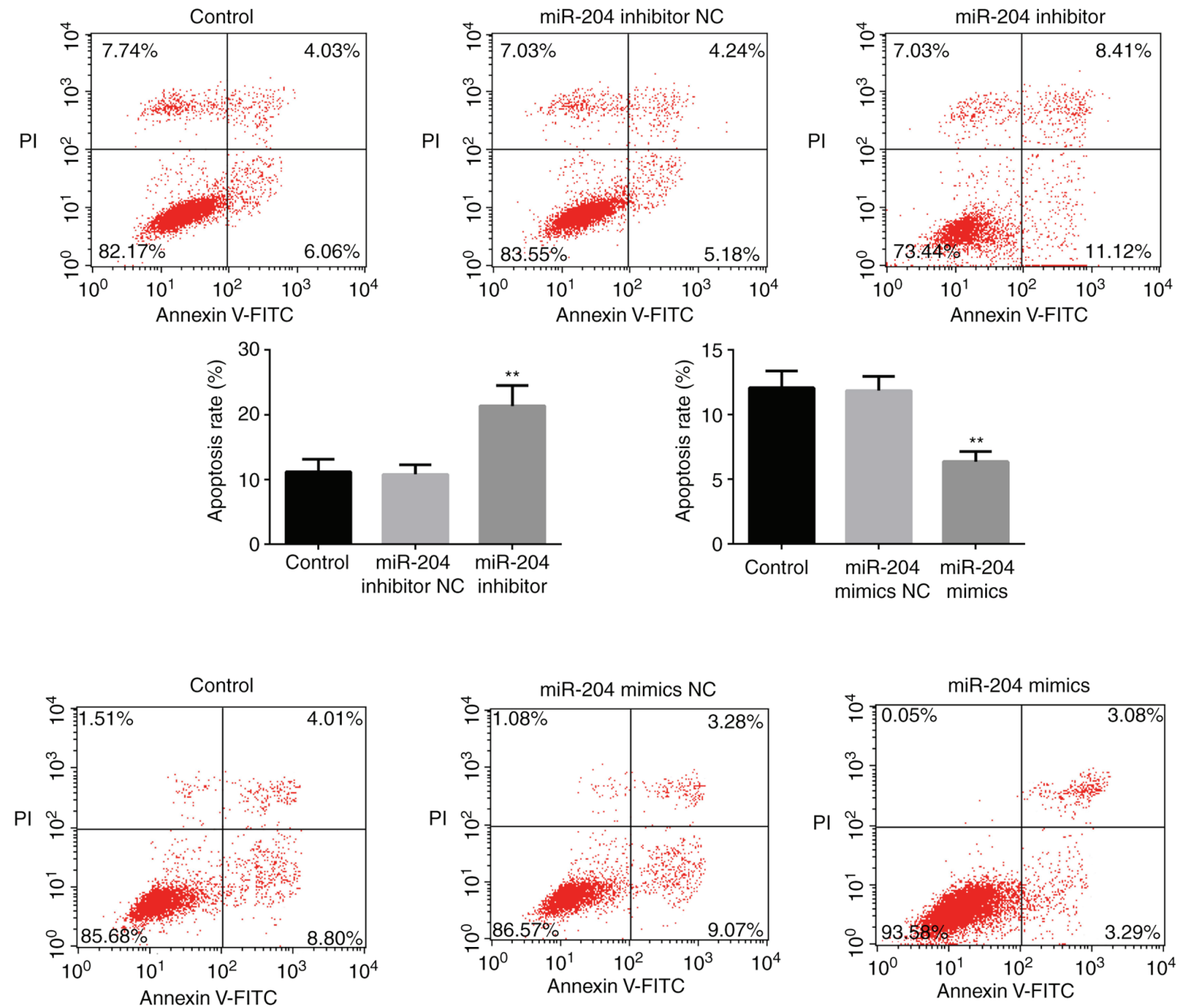

Figure 4. Effect of miR-204 on the apoptosis of neurons at $48 \mathrm{~h}$. Flow cytometry was used to assess apoptosis. ${ }^{* *} \mathrm{P}<0.01$ vs. control. Control, untransfected cells; miR-204, microRNA-204; PI, propidium iodide; FITC, fluorescein isothiocyanate; miR-204 inhibitor, cells transfected with miR-204 inhibitor; miR-204 inhibitors NC, cells transfected with miR-204 inhibitor NC; miR-204 mimics, cells transfected with miR-204 mimics; miR-204 mimics NC, cells transfected with miR-204 mimics NC.

Western blot analysis. Total protein was extracted from rat hippocampal neurons using radioimmunoprecipitation assay buffer (Beyotime Institute of Biotechnology, Shanghai, China). Total protein was quantified using a bicinchoninic acid assay (Beyotime Institute of Biotechnology) and $30 \mu \mathrm{g}$ protein/lane was separated via SDS-PAGE on a $8 \%$ gel. The separated proteins were transferred onto polyvinylidene fluoride membranes and blocked for $1 \mathrm{~h}$ at room temperature with 5\% non-fat milk. The membranes were incubated with primary antibodies against KLLN (1:1,000; cat. no. ab197892) and GAPDH (1:2,000; cat. no. ab9485; both Abcam, Cambridge, MA, USA) overnight at $4^{\circ} \mathrm{C}$. Following primary incubation, membranes were incubated at room temperature for $45 \mathrm{~min}$ with horseradish peroxidase-conjugated secondary antibody (1:2,000; cat. no. ab6721; Abcam). Protein bands were visualized using the enhanced chemiluminescent reagent (Beyotime Institute of Biotechnology) on a ChemiDoc XRS ${ }^{+}$ imaging system (Bio-Rad Laboratories, Inc., Hercules, CA, USA). Protein bands were quantified using ImageJ software (version 1.8, National Institutes of Health) with GAPDH as a loading control.
Cell proliferation assay. The proliferation of the primary rat hippocampus neurons were determined using the MTT Cell Proliferation and Cytotoxicity Assay kit (Beyotime Institute of Biotechnology), according to the manufacture's protocol. Formazan was dissolved in DMSO, and the wavelength used to measure formazan was $570 \mathrm{~nm}$.

Cell apoptosis assay. Following 48-h transfection, cells were subsequently stained with the annexin V-fluorescin isothiocyanate and propidium iodide using the Annexin V Apoptosis Detection kit (BD Biosciences, Franklin Lakes, NJ, USA). Cell apoptosis was analyzed using a BD FACSVerse flow cytometer (BD Biosciences) and FlowJo software (version 7.5.5; Tree Star, Inc., Ashland, OR, USA).

Bioinformatics analysis. The online bioinformatic tool TargetScan (www.targetscan.org/vert_72) was used to predict potential targets of miR-204, as previously described (17). KLLN was identified as a target gene of miR-204. Furthermore, the pairing site at the $3^{\prime}$-UTR of miR-204 and KLLN was determined. 
A Position 2273-2279 of KLLN 3' UTR 5 $5^{\prime}$...GUAGAAGCUUGUAACAAAGGGAC... miR-204-5p 3' UCCGUAUCCUACUGUUUCCCCUU f KLLN $3^{\prime}$ UTR $5^{\prime}$...GUAGAAGCUUGUAACAUAGCCAC..

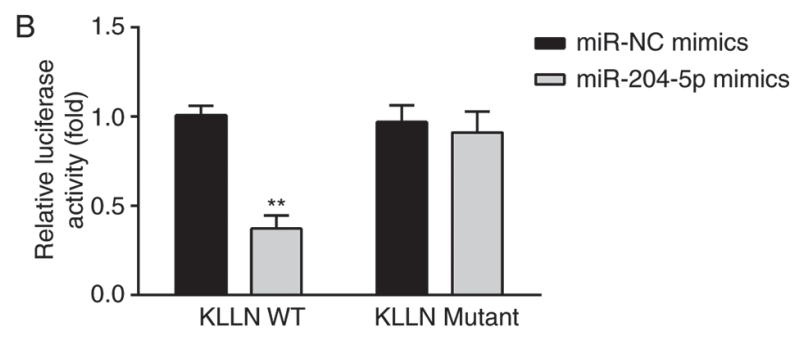

Figure 5. KLLN is a direct target of miR-204 according to dual-luciferase reporter analysis. (A) Sequence alignment of the pairing site at the 3'-UTR of miR-204 and KLLN. (B) Results of the dual-luciferase reporter analysis. ${ }^{* *} \mathrm{P}<0.01$ vs. miR-NC mimics. miR, microRNA; KLLN, killin p53 regulated DNA replication inhibitor; UTR, untranslated region; NC, negative control; KLLN WT, wild-type KLLN-3'-UTR cells; KLLN MT, mutant KLLN-3'-UTR cells; miR-204-5p mimics, cells transfected with miR-204-5p mimics; miR-NC mimics, cells transfected with miR-204-5p mimics NC.

Dual-luciferase reporter assay. The wild-type 3'-UTR of KLLN (KLLN-3'-UTR) and the mutant 3'-UTR of KLLN (KLLN-MUT) region containing the miR-204 binding site were synthesized and cloned into the p-MIR-reporter plasmid (Thermo Fisher Scientific, Inc.). 293 cells were subsequently seeded onto 12 -well plates at a density of $1 \times 10^{6}$ cells/well and transfected with KLLN-3'-UTR or KLLN-MUT and miR-204 mimics or miR-204 mimics NC using Lipofectamine RNAiMax transfection reagent. Following 48-h incubation, cells were collected and luciferase activities were detected using a Dual-Luciferase Reporter system (Beyotime Institute of Biotechnology). Firefly luciferase activity was normalized to Renilla luciferase activity.

Statistics. Statistical analysis was performed using SPSS 19.0 software (IBM Corp., Armonk, NY, USA). Data are presented as the mean \pm standard deviation. The two-tailed Student's t-test was used to compare two groups and one-way analysis of variance with Turkey's post-hoc test was performed for the comparison among multiple groups. $\mathrm{P}<0.05$ was considered to indicate a statistically significant difference.

\section{Results}

Establishing the HIE rat model. To determine the roles of miR-204 in HIE, rat HIE models were established. The infarct size of the brain was measured using a TTC assay. As indicated in Fig. 1A and B, compared with the controls group, the HI-induced brain infarct size was approximately $35 \%$ of the brain (the white part, $0.34 \pm 0.04$ vs. $0.05 \pm 0.01, \mathrm{P}<0.001$ ); furthermore, the results of the TUNEL staining assay indicated that cell death rate in the HIE group was markedly increased compared with the control group (Fig. 1B). Taken together, these results suggested that the HIE model was successfully established.

Decreased expression of miR-204 in the brain tissue of HIE rats. Total RNA was isolated from the brain tissue of HIE rats, and the expression of miR-204 was examined using RT-qPCR. As indicated in Fig. 1C, the expression of miR-204
A

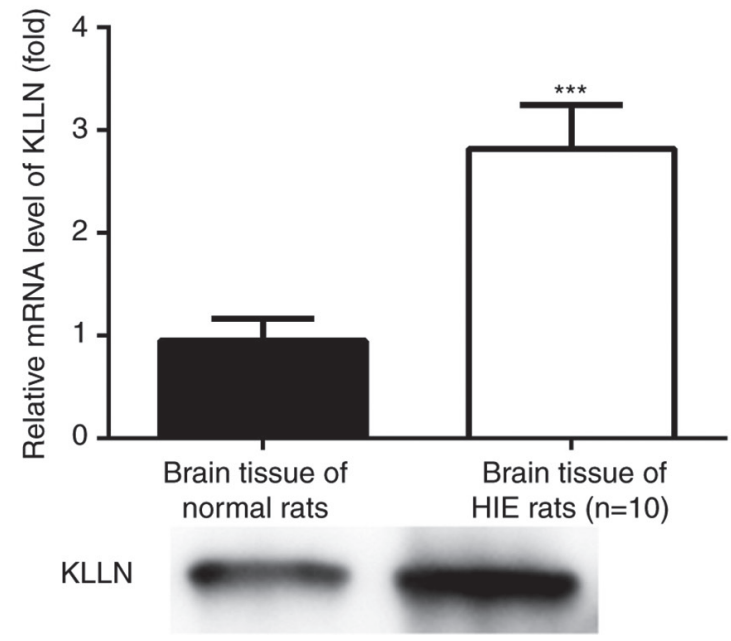

GAPDH
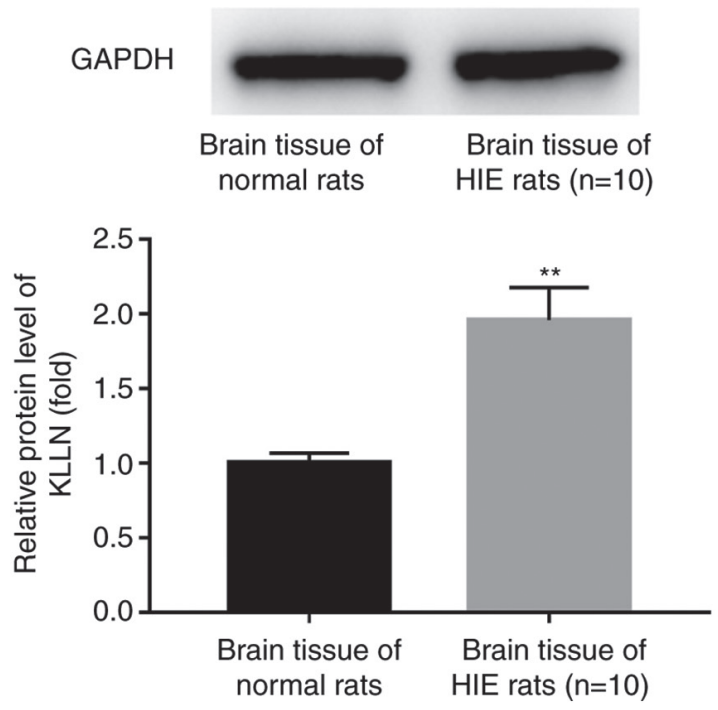

Figure 6. Comparison of the expression of KLLN in HIE and control rats (A) mRNA expression of KLLN in HIE and control rats. (B) Protein expression of KLLN in HIE and control rats. ${ }^{* * *} \mathrm{P}<0.001$ and ${ }^{* *} \mathrm{P}<0.01$ vs. brain tissue of normal rats. miR, microRNA; KLLN, killin p53 regulated DNA replication inhibitor; HIE, hypoxic-ischemic encephalopathy; miR, microRNA.

was significantly downregulated in the brain tissue of HIE rats compared with the control group $(\mathrm{P}<0.001)$.

Effect of miR-204 on the proliferation and apoptosis of neurons. To further explore the roles of miR-204 in the pathogenesis of HIE, neurons were cultured in vitro and transfected with either miR-204 inhibitor or mimics. As indicated in Fig. 2, transfection with miR-204 inhibitor $(20,50$ or $100 \mathrm{nM})$ significantly reduced the expression of miR-204 in neurons, while miR-204 mimics $(20,50$ or $100 \mathrm{nM})$ significantly increased the expression of miR-204 $(\mathrm{P}<0.01$ and $\mathrm{P}<0.001)$. However, miR-204 inhibitor $\mathrm{NC}(20,50$ or $100 \mathrm{nM})$ or miR-204 mimics $\mathrm{NC}$ $(20,50$ or $100 \mathrm{nM})$ had no significant effects on the expression of miR-204 in neurons. Base on the results, $50 \mathrm{nM}$ miR-204 inhibitor or mimics, and $20 \mathrm{nM} \mathrm{miR-204}$ inhibitor NC or mimics $\mathrm{NC}$ were used for the further analysis. Furthermore, the effect of miR-204 on the proliferation and apoptosis of the neurons was examined using MTT and flow cytometry methods. As indicated in Figs. 3 and 4, transfection with miR-204 inhibitors significantly decreased the proliferation rate and increased the 

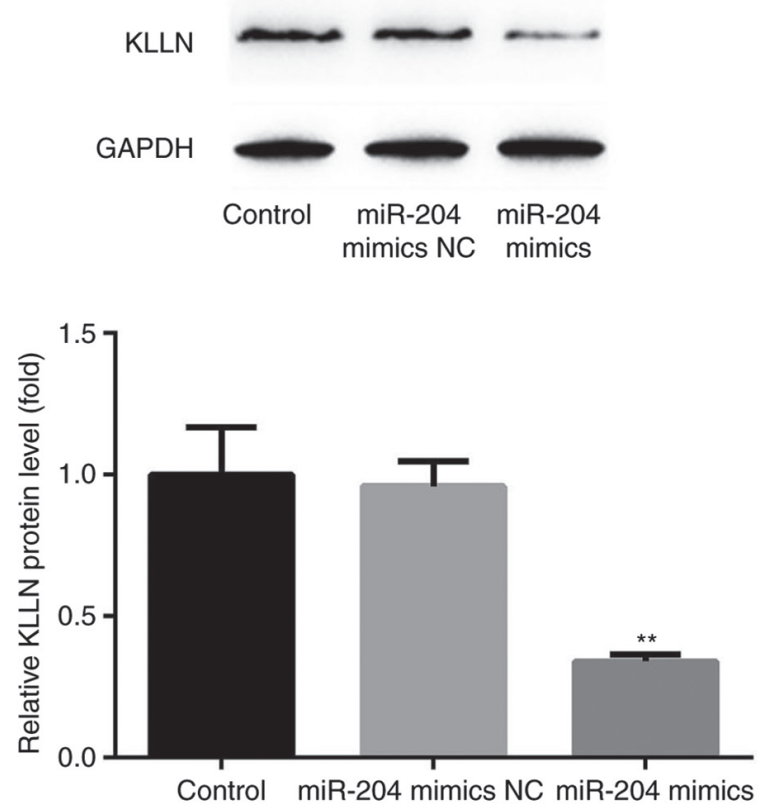

KLLN

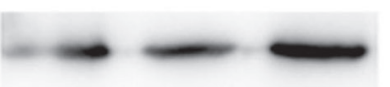

GAPDH

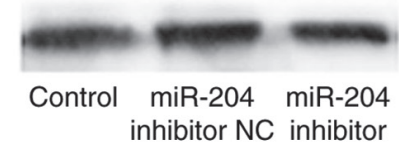

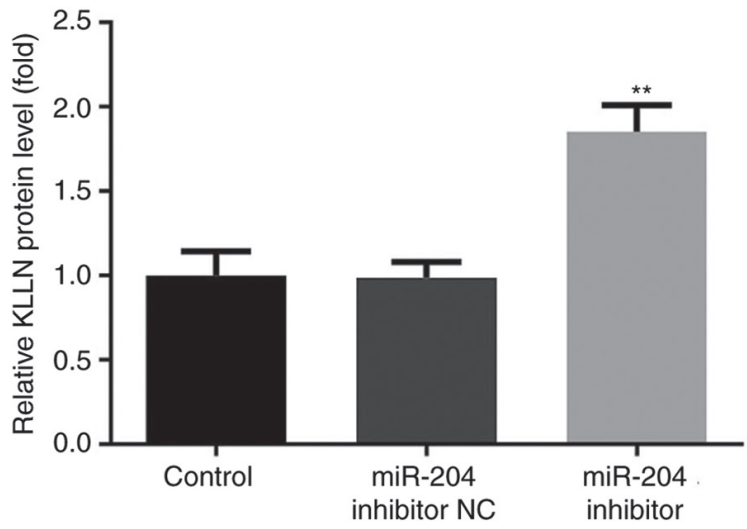

Figure 7. Effect of miR-204 on the expression of KLLN in neurons using western blot analysis. ${ }^{* *} \mathrm{P}<0.01$ vs. Control. Control, untransfected cells; miR, microRNA; KLLN, killin 533 regulated DNA replication inhibitor; NC, negative control; miR-204 inhibitor, cells transfected with miR-204 inhibitor; miR-204 inhibitors NC, cells transfected with miR-204 inhibitor NC; miR-204 mimics, cells transfected with miR-204 mimics; miR-204 mimics NC, cells transfected with miR-204 mimics NC.

apoptosis rate of neurons, whereas transfection with miR-204 mimics resulted in the opposite effects $(\mathrm{P}<0.05$ and $\mathrm{P}<0.01)$.

KLLN is a direct target of miR-204 in neurons. KLLN was predicted to be one of the targets of miR-204 using the online tool TargetScan (Fig. 5A). Based on this, the association between miR-204 and KLLN in the pathogenesis of HIE was explored. Firstly, the results of the dual-luciferase reporter assay indicated that transfection with miR-204 mimics could significantly suppress the activity of the luciferases in wild-type KLLN-3'-UTR cells, whereas miR-204 mimics had no significant effect on KLLN-MUT cells ( $\mathrm{P}<0.01$; Fig. $5 \mathrm{~B})$. This suggested that miR-204 could directly target KLLN. Furthermore, the expression of KLLN in the brain tissue of HIE and normal rats were compared, and it was observed that KLLN expression was significantly increased in HIE models at mRNA and protein levels $(\mathrm{P}<0.01$ and $\mathrm{P}<0.001$; Fig. 6). Notably, the transient knockdown of miR-204 significantly increased the expression of KLLN, whereas overexpression of miR-204 significantly decreased the expression of KLLN in neurons $(\mathrm{P}<0.01$; Fig. 7$)$.

\section{Discussion}

In the present study, the roles of miR-204 and its target gene KLLN in HIE, as well as the underlying mechanism, were investigated using in vivo and in vitro analysis. It was observed that miR-204 was significantly downregulated and KLLN was significantly upregulated in HIE rat models, suggesting that miR-204 may serve important roles in the pathogenesis of HIE through targeting KLLN.

The roles of miRs in the pathogenesis of brain diseases in neonatal rats have been discussed in previous studies. For example, Ma et al (18) reported that miR-210 can inhibit the expression of junction proteins in neonatal rat hypoxic-ischemic brain injury. Furthermore, Wang et al (19) observed that the downregulation of miR-210 can reverse nicotine-induced brain injury in neonatal rats. Looney et al (20) reported that miR-374a was downregulated in the umbilical cord blood of infants with HIE. In addition, Ding et al (21) demonstrated that miR-182 can regulate the expression of pineal clock in HIE rat models. In the present study, it was observed that miR-204 expression was significantly downregulated in the brain tissue of HIE rats, which was consistent with the observation of Qiao et al (12), suggesting that miR-204 may participate in the occurrence and progression of HIE.

It has been reported in previous studies that the HIE condition can lead to increased apoptosis of the neurons in the brain, suggesting that aberrant apoptosis of neurons may have an essential role in the pathogenesis of $\operatorname{HIE}(22,23)$. miR-induced post-transcriptional regulation may have substantial effects on apoptosis-associated pathological and physiological events $(24,25)$. It has been confirmed that miR-204 is involved the proliferation and apoptosis of different type of cells. For example, Shu et al (26) suggested that miR-204 may increase mitochondrial apoptosis in doxorubicin-treated prostate cancer cells via targeting Sirtuin 1 (SIRT1); Zhao et al (27) reported that miR-204 may regulate the proliferation and apoptosis of osteosarcoma cell via SATA3 signaling pathway; and Lin et al (28) identified that miR-204-5p can affect the apoptosis of prostate cancer cells through targeting B-cell lymphoma-2 (BCL-2). Therefore, in the present study, it was postulated that downregulation of miR-204 may increase the apoptosis of neurons and contribute to the pathogenesis of HIE. To confirm the hypothesis of the present study, neuron cells were transfected with miR-204, and it was observed that the transfection 
of miR-204 inhibitors significantly decreased the proliferation rate and increased the apoptosis rate of neurons, whereas transfection with miR-204 mimics produced the opposite effects. Taken together, these results suggested that miR-204 may be involved in the pathogenesis of HIE by affecting the proliferation and apoptosis of neurons in the brain.

Using online bioinformatic tools, KLLN was predicted as a target gene of miR-204. KLLN is a gene that encodes the protein killin, which is a p53-regulated DNA replication inhibitor (29). KLLN is located at the $10 \mathrm{q} 23$ of the chromosome and shares the same transcription start site with PTEN (which is known as a tumor suppressor) (30). The primary function of KLLN has been associated with the regulation of the proliferation and apoptosis of the cells, and overexpression of KLLN can lead to inhibited proliferation and increased apoptosis of the cells, whereas knockdown of KLLN can increase the proliferation and inhibit the apoptosis of different types of cells $(31,32)$. Notably, hypermethylation of KLLN has been demonstrated to participate in the process of Cowden syndrome (33), and the dysregulation of KLLN has been associated with the development of breast cancer (34) and ovarian cancer (35). The roles of KLLN in HIE remain to be investigated. In the present study, as a pro-apoptotic gene, it was hypothesized that downregulation of miR-204 may induce the upregulation of KLLN, which may lead to the aberrant apoptosis of neurons and contribute to the pathogenesis of HIE. The association between miR-204 and KLLN in the pathogenesis of HIE was explored using in vivo and in vitro analysis. The results of the dual-luciferase reporter assay confirmed that miR-204 directly targets KLLN; furthermore, it was observed that the expression of KLLN was significantly upregulated in HIE models in vivo, and in vitro analysis indicated that the downregulation of miR-204 led to increased KLLN expression, whereas upregulation of miR-204 suppressed the expression of KLLN in neurons. Taken together, these results indicated that miR-204 may be involved in the pathogenesis of HIE through targeting KLLN.

It is well known that one miR can have multiple targets. It has been observed that miR-204 can protect $\mathrm{H} 9 \mathrm{C} 2$ cells against hypoxia/reoxygenation-induced injury via regulating SIRT1 induced apoptosis (36); furthermore, miR-204 can promote the apoptosis of prostate cancer cells by targeting BCL-2 (28). SIRT1 and BCL-2 are known anti-apoptotic genes, thus in future studies, the association between miR-204 and SIRT1 or BCL-2 in HIE should be explored.

In conclusion, it was observed that miR-204 was downregulated in HIE. To the best of our knowledge, the present study reported for the first time that miR-204 may serve regulatory roles in the pathogenesis of HIE through targeting KLLN. The results have provided evidence for the application of miR-204 as a novel therapeutic target for the treatment of HIE.

\section{Acknowledgements}

Not applicable.

\section{Funding}

The present study was supported by a grant from the Shenzhen Science and Technology Innovation Committee (grant no. JCYJ20150403091931177).

\section{Availability of data and materials}

The datasets used and/or analyzed during the current study are available from the corresponding author on reasonable request.

\section{Authors' contributions}

RC designed the study. RC, MW and SF performed the experiments. RC, FC and PD performed the experiments, wrote part of the manuscript, and JL analyzed the data.

\section{Ethics approval and consent to participate}

Not applicable.

\section{Patient consent for publication}

Not applicable.

\section{Competing interests}

The authors declare that they have no competing interests.

\section{References}

1. Leroy-Terquem E, Vermersch AI, Dean P, Assaf Z, Boddaert N, Lapillonne A and Magny JF: Abnormal interhemispheric synchrony in neonatal hypoxic-ischemic encephalopathy: A retrospective pilot study. Neonatology 112: 359-364, 2017.

2. Yum SK, Seo YM, Kwun Y, Moon CJ, Youn YA and Sung IK: Therapeutic hypothermia in infants with hypoxic-ischemic encephalopathy and reversible persistent pulmonary hypertension: Short-term hospital outcomes. J Matern Fetal Neonatal Med 31: 3108-3114, 2018.

3. Jain SV, Pagano L, Gillam-Krakauer M, Slaughter JC, Pruthi S and Engelhardt B: Cerebral regional oxygen saturation trends in infants with hypoxic-ischemic encephalopathy. Early Human Dev 113: 55-61, 2017.

4. Ma Q and Zhang L: MiR210 in neonatal hypoxic-ischemic encephalopathy. Oncotarget 8: 38078-38079, 2017.

5. Chen J, Cui C, Yang X, Xu J, Venkat P, Zacharek A, Yu P and Chopp M: MiR-126 affects brain-heart interaction after cerebral ischemic stroke. Transl Stroke Res 8: 374-385, 2017.

6. Wang J, Chen T and Shan G: miR-148b regulates proliferation and differentiation of neural stem cells via $\mathrm{Wnt} / \beta$-catenin signaling in rat ischemic stroke model. Front Cell Neurosci 11: $329,2017$.

7. Wei H, Zhang JJ and Tang QL: MiR-638 inhibits cervical cancer metastasis through Wnt/beta-catenin signaling pathway and correlates with prognosis of cervical cancer patients. Eur Rev Med Pharmacol Sci 21: 5587-5593, 2017.

8. Wu H, Liu HY, Liu WJ, Shi YL and Bao D: miR-377-5p inhibits lung cancer cell proliferation, invasion, and cell cycle progression by targeting AKT1 signaling. J Cell Biochem Nov 28, 2018 (Epub ahead of print).

9. Pan L, Tang Z, Pan L and Tang R: miR-3666 inhibits lung cancer cell proliferation, migration and invasion by targeting BPTF. Biochem Cell Biol 97: 415-422, 2019.

10. Mansoori B, Mohammadi A, Ghasabi M, Shirjang S, Dehghan R, Montazeri V, Holmskov U, Kazemi T, Duijf P, Gjerstorff M and Baradaran B: miR-142-3p as tumor suppressor miRNA in the regulation of tumorigenicity, invasion and migration of human breast cancer by targeting Bach-1 expression. J Cell Physiol 234: 9816-9825, 2019.

11. Yang L, Hou J, Cui XH, Suo LN and Lv YW: MiR-133b regulates the expression of CTGF in epithelial-mesenchymal transition of ovarian cancer. Eur Rev Med Pharmacol Sci 21: 5602-5609, 2017.

12. Qiao Y, Peng C, Li J, Wu D and Wang X: Spinal cord ischemia-reperfusion causes damage of neurocyte by inhibiting RAP2C. Neurol Res 39: 877-884, 2017. 
13. Peng T, Jia YJ, Wen QQ, Guan WJ, Zhao EY and Zhang BA Expression of microRNA in neonatal rats with hypoxic-ischemic brain damage. Zhongguo Dang Dai Er Ke Za Zhi 12: 373-376, 2010 (In Chinese).

14. Edwards AB, Feindel KW, Cross JL, Anderton RS, Clark VW, Knuckey NW and Meloni BP: Modification to the Rice-Vannucci perinatal hypoxic-ischaemic encephalopathy model in the P7 rat improves the reliability of cerebral infarct development after 48 hours. J Neurosci Methods 288: 62-71, 2017.

15. Gonzalez-Rodriguez PJ, Li Y, Martinez F and Zhang L: Dexamethasone protects neonatal hypoxic-ischemic brain injury via L-PGDS-dependent PGD2-DP1-pERK signaling pathway. PLoS One 9: e114470, 2014.

16. Livak KJ and Schmittgen TD: Analysis of relative gene expression data using real-time quantitative PCR and the 2(-Delta Delta C(T)) method. Methods 25: 402-408, 2001.

17. Jin Q, He W, Chen L, Yang Y, Shi K and You Z: MicroRNA-101-3p inhibits proliferation in retinoblastoma cells by targeting EZH2 and HDAC9. Exp Ther Med 16: 1663-1670, 2018.

18. Ma Q, Dasgupta C, Li Y, Huang L and Zhang L: MicroRNA-210 suppresses junction proteins and disrupts blood-brain barrier integrity in neonatal rat hypoxic-ischemic brain injury. Int J Mol Sci 18: E1356, 2017.

19. Wang L, Ke J, Li Y, Ma Q, Dasgupta C, Huang X, Zhang L and Xiao D: Inhibition of miRNA-210 reverses nicotine-induced brain hypoxic-ischemic injury in neonatal rats. Int J Biol Sci 13: 76-84, 2017.

20. Looney AM, Walsh BH, Moloney G, Grenham S, Fagan A, O'Keeffe GW, Clarke G, Cryan JF, Dinan TG, Boylan GB and Murray DM: Downregulation of umbilical cord blood levels of miR-374a in neonatal hypoxic ischemic encephalopathy. J Pediatr 167: 269-273.e2, 2015.

21. Ding X, Sun B, Huang J, Xu L, Pan J, Fang C, Tao Y, Hu S, Li R, Han X, et al: The role of miR-182 in regulating pineal CLOCK expression after hypoxia-ischemia brain injury in neonatal rats. Neurosci Lett 591: 75-80, 2015.

22. Fang C, Xie L, Liu C, Fu C, Ye W, Liu H and Zhang B: Tanshinone IIA improves hypoxic ischemic encephalopathy through TLR-4-mediated NF- $\mathrm{BB}$ signal pathway. Mol Med Rep 18: 1899-1908, 2018.

23. Wang B, Armstrong JS, Lee JH, Bhalala U, Kulikowicz E, Zhang H, Reyes M, Moy N, Spicer D, Zhu J, et al: Rewarming from therapeutic hypothermia induces cortical neuron apoptosis in a swine model of neonatal hypoxic-ischemic encephalopathy. J Cereb Blood Flow Metab 35: 781-793, 2015.

24. Wang CM, Cheng BH, Xue QJ, Chen J and Bai B: MiR-1298 affects cell proliferation and apoptosis in C6 cells by targeting SET domain containing 7. Int J Immunopathol Pharmacol 30: 264-271, 2017.
25. Huang Q, Zheng Y, Ou Y, Xiong H, Yang H, Zhang Z, Chen S and Ye Y: miR-34a/Bcl-2 signaling pathway contributes to age-related hearing loss by modulating hair cell apoptosis. Neurosci Lett 661: 51-56, 2017.

26. Shu Y, Ren L, Xie B, Liang Z and Chen J: MiR-204 enhances mitochondrial apoptosis in doxorubicin-treated prostate cancer cells by targeting SIRT1/p53 pathway. Oncotarget 8: 97313-97322, 2017.

27. Zhao R, He H, Zhu Y, Wan J, Li Y, Gao S and Zhang C: MiR-204/14-3-3 $\zeta$ axis regulates osteosarcoma cell proliferation through SATA3 pathway. Pharmazie 72: 593-598, 2017.

28. Lin YC, Lin JF, Tsai TF, Chou KY, Chen HE and Hwang TI: Tumor suppressor miRNA-204-5p promotes apoptosis by targeting BCL2 in prostate cancer cells. Asian J Surg 40: 396-406, 2017.

29. Sankunny M and Eng C: KLLN-mediated DNA damage-induced apoptosis is associated with regulation of p53 phosphorylation and acetylation in breast cancer cells. Cell Death Dis 4: 31, 2018.

30. Cho YJ and Liang P: Killin is a p53-regulated nuclear inhibitor of DNA synthesis. Proc Natl Acad Sci USA 105: 5396-5401, 2008.

31. Wang Y, He X, Yu Q and Eng C: Androgen receptor-induced tumor suppressor, KLLN, inhibits breast cancer growth and transcriptionally activates p53/p73-mediated apoptosis in breast carcinomas. Hum Mol Genet 22: 2263-2272, 2013.

32. Wang Y, Radhakrishnan D, He X, Peehl DM and Eng C: Transcription factor KLLN inhibits tumor growth by $A R$ suppression, induces apoptosis by TP53/TP73 stimulation in prostate carcinomas, and correlates with cellular differentiation. J Clin Endocrinol Metab 98: E586-E594, 2013.

33. Nizialek EA, Mester JL, Dhiman VK, Smiraglia DJ and Eng C: KLLN epigenotype-phenotype associations in Cowden syndrome. Eur J Hum Genet 23: 1538-1543, 2015.

34. Wang Y, Yu Q, He X, Romigh T, Altemus J and Eng C: Activation of AR sensitizes breast carcinomas to NVP-BEZ235's therapeutic effect mediated by PTEN and KLLN upregulation. Mol Cancer Ther 13: 517-527, 2014.

35. Hu K and Liang M: Upregulated microRNA-224 promotes ovarian cancer cell proliferation by targeting KLLN. In Vitro Cell Dev Biol Anim 53: 149-156, 2017.

36. Qiu R, Li W and Liu Y: MicroRNA-204 protects H9C2 cells against hypoxia/reoxygenation-induced injury through regulating SIRT1-mediated autophagy. Biomed Pharmacother 100: $15-19,2018$

This work is licensed under a Creative Commons Attribution-NonCommercial-NoDerivatives 4.0 International (CC BY-NC-ND 4.0) License. 